\title{
RESSENYES
}

BriLli, Elisa

Firenze e il profeta. Dante fra teologia e politica

Roma: Carocci, 2012, 383 p.

ISBN: 978-8843066568

Fin dai suoi primi saggi, Elisa Brilli ha mostrato di saper reimpostare da capo questioni che per complessità e ampiezza farebbero tremare le vene e i polsi a molti studiosi (mi limito a citare l'ottimo "Dalla "città partita" alla "civitas confusionis". Sulla tradizione e i modelli della Firenze dantesca», in Bollettino di Italianistica, n.s. III, 1, 2006, p. 73-111, in cui alcuni dei contenuti del libro che si va esponendo sono precocemente anticipati). Firenze e il profeta si pone su questa scia, riacquisendo e collocando in un corpo interpretativo articolato alcuni dei temi di ricerca già affrontati dall'autrice: se ne ricava un lavoro pregevole, i cui risultati - frutto, con ogni evidenza, di un lungo pensiero- promuovono uno sguardo nuovo su problemi da sempre centrali negli studi danteschi, ma raramente affrontati in modo altrettanto sistematico, e con eguale respiro. Si tratta infatti di ricostruire l'immagine di Firenze restituita nei testi dell'esilio: ciò allo scopo di vagliare da un punto di vista quanto più ampio e documentato il peso della storia fiorentina nel divenire del pensiero politico e teologico di Dante, e nella rappresentazione che il poeta offre progressivamente di sé - questi gli obiettivi della monografia (esposti in estrema sintesi, s'intende). Brilli entra dunque nel vivo di almeno due degli snodi concettualmente più impervi dell'esperienza poetica dantesca: il ripensamento della storia municipale alla luce dell'esilio e la vocazione profetica; e tra di essi individua un notevole legame. L'indagine, pur accordata a una struttura ricorrente - in cui alla preliminare esposizione di quadri teorici complessi segue, con rigore, il momento dell'esegesi testuale_-, non appare mai schematica: al contrario, la ripresa e il chiarimento a distanza di determinati nuclei concettuali conferiscono al libro un andamento assai vivace. Così, la selezione dei materiali fiorentini operata da Dante nella Commedia, sostanzialmente adeguata ai punti fermi della memoria municipale per gli eventi anteriori al 1280 (è quanto si ricostruisce nel cap. 1), assume un senso pieno alla luce dell'incontro con Cacciaguida, che esorta il discendente a narrare il suo viaggio privilegiando le vicende delle "anime che son di fama note» (Paradiso, XvII 138); 
la definitiva investitura cacciaguidiana, che sancisce l'assunzione di un modello auto-rappresentativo di tipo apostolicoprofetico (cap. 3), trova il suo motivo fondamentale nel rapporto con la città antagonista, Firenze, progressivamente riconosciuta come civitas diaboli dall'esiliato che ripensa la storia del comune (cap. 1-2). Ed è quanto meno plausibile - spiega Brilli, chiudendo il cerchio - che in Dante "proprio la spinosissima materia fiorentina sollecitasse per prima il ricorso a strumenti testuali scritturali e profetico-apocalittici perché più efficaci in vista della costruzione e del riscatto di sé» (cap. 3; p. 304). Il corpo centrale del libro, occupato dal capitolo secondo, costituisce con ogni probabilità il nucleo scientifico più complesso e originale della ricerca, oltre che un momento decisivo per tutta la sua struttura argomentativa (La «civitas diaboli sub specie Florentiae», p. 121-270). Notevole è già la sezione iniziale (p. 121-127), in cui l'autrice, sintetizzando i risultati della sua tesi di dottorato (Una vicina città. Storia del paradigma della "civitas diaboli" nell'Occidente medievale, di prossima pubblicazione), riesce a fornire in pochi paragrafi una mappa dei sensi veicolati dalla formula civitas diaboli senza occultare gli esiti più ardui che questa figura, nel suo concreto articolarsi ideologico, produce. La civitas diaboli designa «la compagine metastorica degli empi» (p. 123): è perciò un paradigma, che la teologia agostiniano-medievale della storia invita a riconoscere come costantemente in atto; non un'idea archetipica, un'immagine di sintesi concettuale - teologico-filosofica, e poi poetica. La formula civitas diaboli permette dunque, in una prima accezione, di identificare ogni comunità che abbia storicamente disconosciuto Dio, pretendendo di esaurire nella dimensione del mondo terreno "quella perfetta felicità che il vero cristiano sa di poter attingere solo nell'altro" (ibid.). Ma già nell'espressione civitas terrena, le cui occorrenze mostrano un uso sinonimico rispetto a civitas diaboli, si manifesta un valore decisamente più ampio: «nei discorsi medievali, la civitas diaboli può vedere la propria portata estendersi fino a coincidere con il transeunte e lo storico in quanto tali, con l'(hoc) saeculum distinto e contrapposto a quello a venire a causa della sua strutturale imperfezione» (p. 126). Brilli entra allora nel vivo della contraddizione che pare insinuarsi - per così dire - tra l'accezione particolare e quella universale del paradigma (p. 126127), e la risolve in una nuova, acuta lettura del canto xvi Paradiso (v. 73-87): nel discorso di Cacciaguida, la rassegna delle famiglie fiorentine decadute esemplifica le tragiche conseguenze della confusion delle persone e al contempo «illustra il divenire inesausto delle cose mondane» (p. 238); Firenze viene quindi a configurarsi come civitas diaboli in entrambi i sensi, particolare e universale: sia in quanto caterva impiorum, cioè, che in quanto saeculum obbligatoriamente sottoposto ai rovesci della storia (p. 237 239). Lo spunto non si esaurisce qui: nelle ultime pagine del suo lavoro, Brilli proporrà una soluzione al problema della "doppia autobiografia dantesca» seguendo una pista analoga, e altrettanto persuasiva (3.9, p. 352-354, su cui tornere$\mathrm{mo})$. Al centro del capitolo finale ( $\mathrm{Il}$ profeta «sub specie Dantis», p. 271-354) è il divenire dell'auto-rappresentazione di Dante: dal modello boeziano del saggio capace di contegno davanti ai rovesci della Fortuna (3.3, p. 282-296), perfettamente attivo nei testi dei primi anni dell'esilio (Convivio, I in 13; De vulgari eloquentia, I vi 3...), alla progressiva assunzione di una "maschera profetica» messa in scena nella Commedia. Motivo fondamentale di questo slittamento è il volgersi del pensiero dantesco su Firenze: se il filone fiorentino è il banco di prova su cui la retorica visionaria del poema, preferibilmente, si tempra (p. 300-304), proprio l'antagonismo tra l'exul inmeritus 
e la città — cioè il progressivo riconoscimento di Firenze come occorrenza storica della civitas diaboli- si può intendere come chiave d'innesto della svolta profetica ("la civitas diaboli si conferma come tale per il fatto di accanirsi contro i giusti; a sua volta, il giusto è certificato per il fatto di essere l'oggetto dell'accanimento persecutorio della città e, eletto profeta, di essere incaricato di vaticinarne la punizione divina»; p. 321). Ne consegue un circolo "rappresentativo" pienamente virtuoso: il nesso che stringe Firenze e Dante nella Commedia si manifesta, a livello extra-testuale, nell'ingiustizia dell'esilio; a livello intra-testuale, nel diabolico atteggiamento con cui Firenze, «referente primario della militia profetico-apostolica dantesca» (p. 322), convalida l'investitura dell'autore. Brilli sa bene, per altro, che «in una prospettiva scientifica» il problema del profetismo di Dante "può apparire insolubile» (così Giorgio Inglese, Guida alla "Divina Commedia", Roma: Carocci, 2002, p. 47); perciò la sua indagine — pur debitrice del contributo fondatore di Nardi e dei più recenti studi sulla semiotica e sulla stilistica del discorso profetico di Dante (si vedano le p. 298-300 e la bibliografia citata alle note 36-40) - si orienta opportunamente sui soli modi di rappresentazione («sul senso e sulle forme»; p. 300), evitando interrogativi destinati a restare privi di risposta (la Commedia può effettivamente derivare da un'esperienza di tenore mistico, da un'autentica visione?). Si delinea pertanto anche qui, in questo snodo essenziale del discorso, un quadro perfettamente solido, e del tutto ricevibile su un piano scientifico. Firenze e il profeta si chiude sul problema della doppia autobiografia dantesca (3.7-9, p. 324-354) — sulla relazione conflittuale che tende a insinuarsi tra il racconto «scritto con la penna di Beatrice e intonato al motivo del "traviamento"» di Dante e quello «scritto con la penna di Cacciaguida» e ispirato al motivo della sua gran- dezza "civile e morale» (le espressioni sono di Gennaro SAsso, Le autobiografie di Dante, Napoli: Bibliopolis, 2008, p. 13, da cui Brilli muove esplicitamente la sua riflessione). L'autrice esclude, innanzitutto, che la tradizionale lettura in chiave allegorica dell'esilio, di cui la Commedia costituirebbe l'esito trasfigurato, possa considerarsi sostenibile: lo schema manca di sostegno logico, dato che il viaggio consegnato al senso letterale del poema si pone in un tempo antecedente a quello dell'esilio vero e proprio. Ma è soprattutto la forza con cui Dante dichiara ingiusta la pena a lui comminata da Firenze a impedire ogni movimento allegorico nella direzione dell'exilium umano dalla patria celeste; e come spiega egregiamente Brilli (si leggano le p. 332333), le occasionali affinità di tono tra $i$ temi in gioco —esilio storico, viaggio nell'aldilà, peregrinatio dell'umanità verso Dio- producono tutt'al più un'armonia: non la trasposizione allegorica dell'uno nell'altro. La discrepanza individuata da Sasso non può dunque, né deve risolversi. E tuttavia, considerando le autobiografie dantesche su un piano diacronico - e qui Brilli, di nuovo, si presta proficuamente a rileggere tutto da capole cose in parte cambiano: è indubbia, ad esempio, la priorità del motivo biografico della peregrinatio, ampiamente sfruttato nella Vita nova e ancora attivo nei primi canti della Commedia. Ma il progressivo innestarsi di episodi personali nello schema originario del movimento di conversione, in sé non ingiustificato, subisce un mutamento di prospettiva all'altezza dell'incontro con Brunetto Latini: qui Dante è detto giusto, già prima del viaggio e dell'esilio (Inferno, xv 64). Cosa determinò questo cambio di direzione? Forse proprio il divenire della pratica testuale - è questa, credo, l'intuizione radicale del libro-: la scoperta della formulazione profetica come «strumento più atto a veicolare i messaggi, le speranze e le minacce, che Dante voleva desti- 
nare al proprio pubblico» (p. 343). Non essendo praticabile una riscrittura degli eventi biografici precocemente affidati alla Commedia, poiché il poema sta già circolando, Dante tenta un difficile accordo tra l'autobiografia profetica e quella, originaria, di conversione. Non tutto può quadrare: se il nuovo atteggiamento di Beatrice dopo l'incontro con Cacciaguida non crea «increspature evidenti» sulla superficie del poema (p. 345), e se l'investitura dell'avo consente la piena affermazione dell'autobiografia fiorentina sul modello alternativo dell' $i$ ter di affrancamento dal peccato (di cui questa era originariamente parte), non si annulla la discontinuità ideologica con l'impianto «scritturale, fortemente in debito con i libri profetici» dei primi canti dell, Inferno (p. 346-347). Ma i due paradigmi dissonanti, quello del peccatore in via di conversione e quello del profeta perseguitato, non coabitano solo in Dante: Brilli chiude il suo lavoro ricordando l'analogo meccanismo messo in atto nell' Historia calamitatum di Pietro Albelardo (p. 347-349) e -riscontro notevolissimo- nell'Iconologia overo

\section{La Veneciana}

Madrid: Cátedra, 2013, 158 p. ISBN: 978-84-376-3086-1

Cesáreo Calvo Rigual y Anna Giordano Gramegna editan por primera vez en español La Venexiana, una comedia anónima escrita, según afirma gran parte de la crítica, entre 1535 y 1537 , derivada quizás de un suceso real, y ambientada en una Venecia descrita con minucioso realismo. La comedia se inserta en la corriente del teatro humanista que germinaba y se extendía con pujanza en la Venecia del Renacimiento. Nuevas for-
Descrittione dell'imagini universali cavate dall'antichità et da altri luoghi dell'accademico degli Intronati Cesare Ripa, morto nel 1622 (!). Qui, alla voce esilio, la dualità pubblico/privato è vertiginosamente sintetizzata nella medesima categoria: esiliato è sia il condannato all'espulsione dal potere politico che il viandante diretto a Santiago a scopo penitenziale (p. 349-351). Tra il pellegrino convertito e il giusto perseguitato la cultura dell'Occidente medievale ha dunque riconosciuto un'affinità: e in fondo, se la civitas diaboli è al contempo la caterva impiorum e il saeculum umano, l'allontanamento da essa può leggersi come processo di affrancamento dal peccato e, senza che la dissonanza diventi contraddizione, come riconoscimento di un'investitura profetica (p. 352-354). Ciò dimostra, in definitiva, il percorso di Dante; e ciò illustra, con felicità di pensiero e scrittura, lo studio di Elisa Brilli.

\section{Luca Fiorentini}

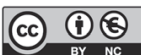

mas teatrales que se desarrollaron desde un plano popular y dialectal con la intención de enseñar y divertir, y que generaron un teatro abierto a la innovación y a la experimentación literaria.

Venecia se presenta, en aquellos años, como un importante centro urbano expuesto a un cosmopolitismo profuso que oxigena de manera constante los usos y costumbres de la vida pública y privada de sus ciudadanos. Y es esta renovación 